\section{Signalisation dans les lymphocytes T Implication de canaux calciques}

Marilena Djata Cabral, Bruno Gomes, Magali Savignac, Marc Moreau, Catherine Leclerc, Lucette Pelletier
Calcium et régulation Calciums normales et pathologiques
> La reconnaissance du peptide antigénique par son récepteur (TCR) entraîne une augmentation de la concentration de $\mathrm{Ca}^{2+}$ dans le cytosol $\left(\left[\mathrm{Ca}^{2+}\right] \mathrm{i}\right)$, nécessaire à la différenciation et aux fonctions effectrices des lymphocytes (L)T. La stimulation via le TCR permet le recrutement de molécules adaptatrices et le couplage à des enzymes, dont la phospholipase $c \gamma$, qui produit de l'inositol 1,4,5 tri-phosphate (IP3) et du diacylglycérol. L'IP3 en se fixant à ses récepteurs du réticulum endoplasmique libère les stocks de $\mathrm{Ca}^{2+}$ dans le cytosol (Figure I). L'entrée de $\mathrm{Ca}^{2+}$ à partir du milieu extracellulaire reconstitue ces stocks et maintient une signalisation soutenue. Les canaux calciques responsables sont définis comme des SOC

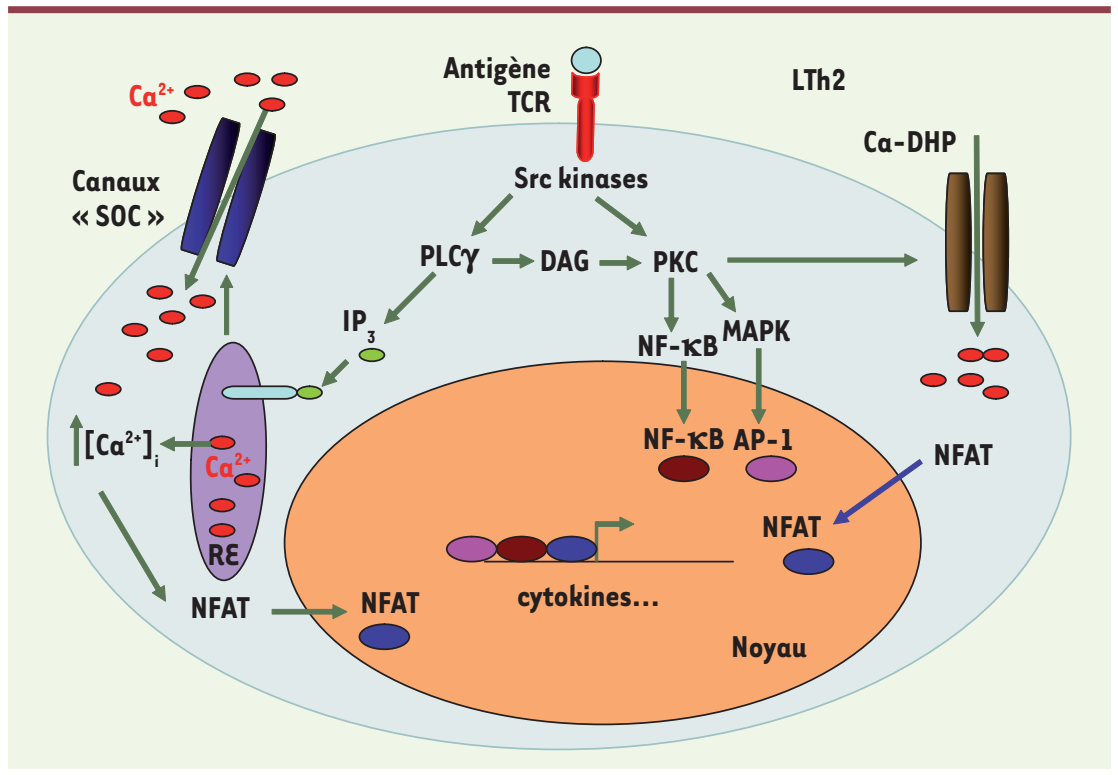

Figure 1. Signalisation calcique dans les lymphocytes T. La stimulation du TCR induit l'activation de la PLC $\gamma$ qui produit de I'IP-3 et du diacylglycérol (DAG). L'IP3, en se fixant à des récepteurs du réticulum endoplasmique (RE), libère les stocks de $\mathrm{Ca}^{2+} \mathrm{du} R \varepsilon$, ce qui active des canaux dépendant des stocks (SOC) à la membrane plasmique. S'ensuit un influx de $\mathrm{Ca}^{2+}$ cytoplasmique nécessaire pour la pleine activation du LT. La principale cible du $\mathrm{Ca}^{2+}$ est la phosphatase calcineurine qui déphosphoryle le facteur de transcription NFAT permettant sa localisation nucléaire. La stimulation via le TCR engage d'autres voies dont celles de la PKC et des MAPK (mitogen activated protein kinase) qui activent des facteurs de transcription tels qu'AP- 1 et NF- $K B$. L'ensemble de ces facteurs de transcription active les gènes cibles dont les gènes codant les cytokines. À côté de ces voies, nous avons montré le rôle de canaux calciques sensibles aux DHP (dihydropyridines) (Ca-DHP), probablement apparentés aux canaux Cavl, impliqués dans l'influx calcique nécessaire à l'activation des LTh2.

M. Djata Cabral, B. Gomes, M. Savignac, L. Pelletier : Inserm U563, CPTP IFR30, CHU Purpan, 1, place du Docteur Baylac, 31059 Toulouse Cedex 9, France.

M. Moreau, C. Leclerc: CNRS, UMR 5547, Centre de Biologie du Développement, Université Paul Sabatier, Toulouse et GDR 2688, Toulouse, France. Lucette.Pelletier@purpan.inserm.fr

(store-operated $\mathrm{Ca}^{2+}$ channels) mais leur identité moléculaire n'est pas complètement élucidée. Un des principaux facteurs de transcription activé par la voie calcique est NFAT (nuclear factor of activated $T$ cells). La calcineurine, une phosphatase régulée par le $\mathrm{Ca}^{2+}$ déphosphoryle NFAT, ce qui permet sa localisation nucléaire et l'expression des gènes cibles.

\section{Hétérogénéité de la signalisation calcique dans les LT}

Classiquement, la description des voies de signalisation associées au TCR ne tient pas compte de l'hétérogénéité des LT qui incluent les LThl et Th2. Les LThl produisent de l'interféron $\gamma$ (IFN $\gamma$ ), du TNF $\alpha$ (tumor necrosis factor $\alpha$ ), tandis que les LTh2 sécrètent les interleukines-4,-5,-13 (IL-4, IL-5, IL-13). Les LThI permettent I'élimination de pathogènes intracellulaires, mais sont associés au développement de maladies auto-immunes. Les LTh2 sont impliqués dans l'éradication des parasites [3], mais causent des maladies allergiques comme l'asthme [1]. La régulation de l'homéostasie calcique diffère dans les LTh1 et Th2. La $\left[\mathrm{Ca}^{2+}\right] \mathrm{i}$ est constitutivement plus élevée dans les LTh2 que dans les LThl, mais la $\left[\mathrm{Ca}^{2+}\right] \mathrm{i}$ s'élève moins dans les LTh2 que dans les LThl après stimulation [2]. Notre groupe a montré que la différenciation des LTh2 - mais pas celle des LThl - s'accompagnait d'une augmentation de l'expression de canaux calciques, sensibles aux DHP (dihydropyridines) (Ca-DHP), vraisemblablement apparentés aux canaux dépendant du voltage (Cavl) des cellules excitables 
[7]. Nous décrirons donc brièvement ces canaux et discuterons de leur présence et

\section{Canaux calciques Cav1}

Les canaux Cavl, sélectifs pour le $\mathrm{Ca}^{2+}$ sont définis comme des récepteurs de la dihydropyridine (RDHP). Ils sont activés dans les cellules excitables, à la suite d'une forte dépolarisation membranaire, comme celle qui engendre un potentiel d'action. Les canaux sont constitués par les sous-unités $\alpha$ l qui forment le pore calcique et des sous-unités auxiliaires qui modulent l'expression du canal à la membrane plasmique, et ses propriétés (Figure 2). Quatre gènes codent les sous-unités $\alpha 1$ (de Cavl.l à Cavl.4), et contrôlent l'expression tissulaire: Cavl.1 prédomine dans le muscle strié, Cavl.2 dans le coeur, le muscle lisse, les neurones, les vaisseaux, Cavl.3 dans les neurones et tissus neuroendocriniens, et Cavl.4 dans la rétine.

\section{Présence de canaux apparentés aux canaux Cavl dans les LT}

Des ARN messagers codant les sousunités $\alpha 1$ des canaux Cavl.2, Cavl.3 [8] et Cav1.4 [4] ont été détectés dans des LT humains après stimulation via le TCR. L'analyse de la taille des protéide leur rôle dans les LT.

nes correspondantes suggérait que leur structure différait de celles présentes dans les cellules excitables. Un agoniste des RDHP induisait une augmentation faible mais soutenue de la $\left[\mathrm{Ca}^{2+}\right]$ i et un antagoniste diminuait la signalisation calcique, la production d'IL-2 et la prolifération des LT. Toutefois, les doses d'antagonistes efficaces étaient très supérieures à celles normalement utilisées pour bloquer les canaux Cavl des cellules excitables, posant le problème de la spécificité de l'effet de l'antagoniste.

\section{Expression préférentielle}

\section{de canaux Cavl au cours}

\section{de la différenciation en LTh2}

Nous avons montré que la différenciation des LTh2, mais pas celle des LThl, s'accompagne d'une augmentation des ARN messagers codant les sous-unités $\alpha 1$ des canaux Cavl.2 et Cavl.3, ce qui est en accord avec la détection des protéines dans les LTh2 mais pas dans les LThl. Un agoniste des RDHP induit une réponse calcique et l'expression de I'IL-4, tandis qu'un antagoniste des RDHP bloque la signalisation calcique et la synthèse d'IL-4. Ces agents n'ont aucun effet sur des LThl. Des antagonistes des RDHP comme la nicardipine sont

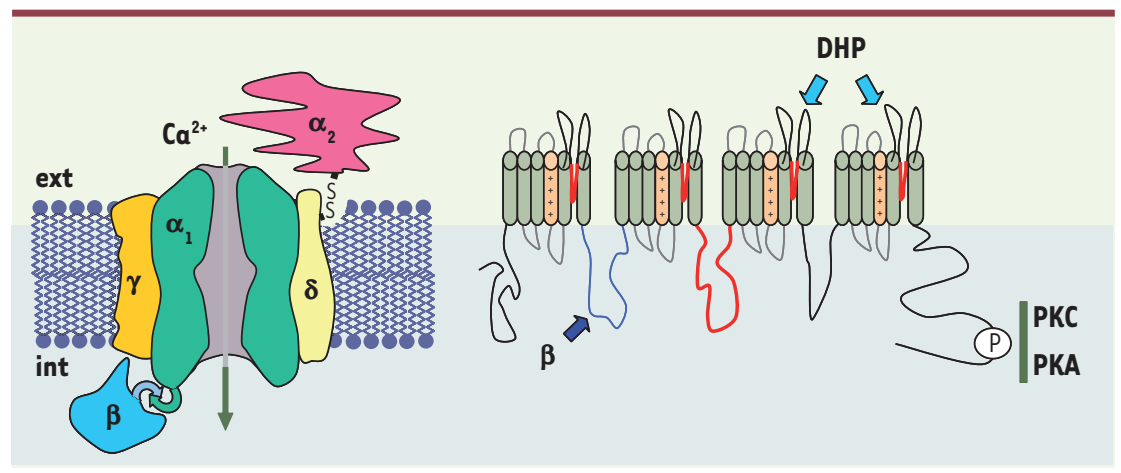

Figure 2. Structure schématique des canaux calciques voltage-dépendants de type 1 (Cav1). A. Description de la structure d'un canal Cavl qui est formé par les sous-unités $\alpha 1, \alpha 2-\delta, \beta$ et $\gamma$. La sous-unité $\beta$ est une protéine cytoplasmique. $B$. La sous-unité $\alpha$ l forme le pore calcique. Cette sous-unité est composée de quatre domaines comprenant chacun six segments. Les segments $\$ 4$ se comportent comme des capteurs du potentiel, ce qui est lié à des séquences d'acides aminés chargés positivement. La boucle intracellulaire I-II des sous-unités $\alpha$ l permet l'interaction avec la sous-unité $\beta$. Les sites de phosphorylation (P) pour les protéine kinases PKA et PKC, localisés dans la partie carboxy-terminale, sont indiqués. couramment utilisés dans le traitement de l'hypertension artérielle et de l'insuffisance cardiaque. L'administration in vivo de nicardipine, un antagoniste des RDHP prévient des pathologies liées aux LTh2 [7], y compris l'asthme. Ainsi, nous avons montré dans des modèles expérimentaux d'asthme que la nicardipine bloque l'infiltration pulmonaire par les LT et les éosinophiles et inhibe la synthèse d'IL-4 par les LTh2. De plus, la transfection de LTh2 avec des oligonucléotides anti-sens spécifiques des canaux Cavl.2 et Cavl.3 inhibe leur expression, la réponse calcique et la capacité de ces LTh2 à transférer l'asthme. Ces données suggèrent fortement le rôle spécifique des canaux Cavl dans les LTh2.

\section{Modèles pouvant expliquer \\ le fonctionnement \\ de ces canaux dans les LT}

Une question non résolue concerne le mode de fonctionnement de ces canaux qui ne sont pas activés par la dépolarisation membranaire dans les LT. Kotturi [4] et Stokes [8] ont montré la présence de protéines tronquées dans les LT, laissant penser qu'une différence de structure par rapport à celle des canaux Cavl des cellules excitables pourrait rendre compte des différences de propriétés électrophysiologiques. Nos données suggèrent la présence de sous-unités $\alpha$ l ayant la même taille dans les LTh2 que dans les cellules excitables. Nous sommes en train de séquencer complètement les ADN complémentaires codant ces sous-unités pour pouvoir les exprimer et étudier leurs propriétés électrophysiologiques. Si ces sous-unités ne sont pas régulées par le potentiel, elles pourraient l'être par phosphorylation. Récemment, Navedo a montré qu'une part importante de la régulation de l'homéostasie calcique dans les cellules artérielles musculaires était due à de rares canaux Cavl constitutivement actifs, car probablement directement couplés à la protéine kinase C (PKC) [5]. Les ions calciques passant au travers de ces canaux pourraient moduler de façon importante la fonction de protéines de voisinage 
sensibles au $\mathrm{Ca}^{2+}$ et impliquées dans de multiples voies de signalisation. De plus, le regroupement de ces canaux pourrait directement influer sur la nature du signal calcique. Si ce concept est applicable aux LT, l'induction du regroupement de canaux après stimulation via le TCR dans des zones de signalisation comme la synapse immunologique et leur couplage à la PKC pourraient réguler l'influx de $\mathrm{Ca}^{2+}$ dépendant des canaux Cavl. Il est à noter que nous avons montré que la PKC contrôlait I'influx calcique via les canaux Ca-DHP dans les LTh2 (Figure 1) [6].

\section{Conclusions}

S'il s'avère que les canaux Cavl jouent un rôle important dans les LTh2 et pas les LThl, l'utilisation d'antagonistes de ces canaux pourrait s'avérer une alternative intéressante dans le traitement des maladies allergiques dont la fréquence ne cesse de croître dans les pays industrialisés. $\diamond$

A role for voltage-dependent related $\mathrm{Ca}^{2+}$ channels in calcium signaling of $\mathrm{T}$ lymphocytes

\section{REMERCIEMENTS}

Ce travail a été réalisé grâce au financement de l'Association de Recherche sur la Polyarthrite Rhumatoïde, de l'Association de Recherche contre le Cancer de la Ligue contre le Cancer et de la Fondation Calouste Gulbenkian.

\section{RÉFÉRENCES}

1. Cohn L, Elias JA, Chupp GL. Asthma: mechanisms of disease persistence and progression. Annu Rev Immunol $2004 ; 22$ : 789-815.

2. Fanger CM, Neben AL, Cahalan MD. Differential $\mathrm{Ca}^{2+}$ influx, $\mathrm{KCa}$ channel activity, and $\mathrm{Ca}^{2+}$ clearance distinguish Th1 and Th2 lymphocytes. J Immunol 2000; $164: 1153-60$

3. Ho IC, Glimcher LH. Transcription: tantalizing times for T cells. Cell $2002 ; 109$ : S109-20.

4. Kotturi MF, Jefferies WA. Molecular characterization of L-type calcium channel splice variants expressed in human T lymphocytes. Mol Immunol 2005 ; 42 : 1461-74.

5. Navedo MF, Amberg GC, Votaw VS, Santana LF. Constitutively active L-type $\mathrm{Ca}^{2+}$ channels. Proc Natl Acad Sci USA 2005 ; 102 : 11112-7.

6. Savignac M, Badou A, Moreau M, Leclerc C, Guery JC, Paulet P, Druet P, Ragab-Thomas J, Pelletier L. Protein kinase $\mathrm{C}$-mediated calcium entry dependent upon dihydropyridine sensitive channels: a T cell receptorcoupled signaling pathway involved in IL-4 synthesis. Faseb J 2001 ; 15 : 1577-9.

7. Savignac M, Gomes B, Gallard A, et al. Dihydropyridine receptors are selective markers of Th2 cells and can be targeted to prevent Th2-dependent immunopathological disorders. J Immunol 2004 ; $172:$ 5206-12.

8. Stokes L, Gordon J, Grafton G. Non-voltage-gated $L$-type $\mathrm{Ca}^{2+}$ channels in human T cells: pharmacology and molecular characterization of the major alpha pore-forming and auxiliary beta-subunits. J Biol Chem $2004 ; 279$ : 19566-73.
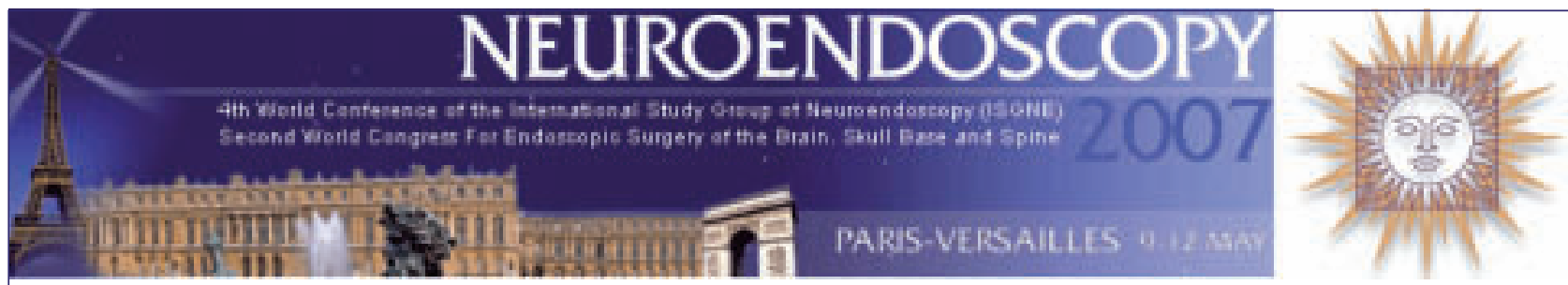

Congrès:

NEUROENDOSCOPY 2007

Dates:

9 - 12 MAI 2007

Lieu:

Paris, Versailles, FRANCE

Secrétariat du congrès:

\section{NEUROENDOSCOPY 2007 c/o MCI France}

24 rue Chauchat - 75009 Paris - FRANCE

Téléphone: +33 (1) 53858253 - Fax: +33 (1) 53858283

Email: neuroendo2007info@mci-group.com

Thèmes:

hydrocéphales

tumeurs ventriculaires

l'approche Endoscopique de base de crâne

\section{www. neuroendoscopy2007.com}

